\title{
Metric Dimension and Exchange Property for Resolving Sets in Rotationally-Symmetric Graphs
}

Rishi Naeem and Muhammad Imran*

Department of Mathematics, School of Natural Sciences (SNS), National University of Sciences and Technology (NUST), Sector H-12, Islamabad, Pakistan

Received: 23 Jul. 2013, Revised: 25 Oct. 2013, Accepted: 26 Oct. 2013

Published online: 1 Jul. 2014

\begin{abstract}
Metric dimension or location number is a generalization of affine dimension to arbitrary metric spaces (provided a resolving set exists). Let $\mathscr{F}$ be a family of connected graphs $G_{n}: \mathscr{F}=\left(G_{n}\right)_{n \geq 1}$ depending on $n$ as follows: the order $|V(G)|=\varphi(n)$ and $\lim _{n \rightarrow \infty} \varphi(n)=\infty$. If there exists a constant $C>0$ such that $\operatorname{dim}\left(G_{n}\right) \leq C$ for every $n \geq 1$ then we shall say that $\mathscr{F}$ has bounded metric dimension, otherwise $\mathscr{F}$ has unbounded metric dimension. If all graphs in $\mathscr{F}$ have the same metric dimension (which does not depend on $n), \mathscr{F}$ is called a family with constant metric dimension.

In this paper, we study the metric dimension of quasi flower snarks, generalized antiprism and cartesian product of square cycle and path. We prove that these classes of graphs have constant or bounded metric dimension. It is natural to ask for characterization of graphs classes with respect to the nature of their metric dimension. It is also shown that the exchange property of the bases in a vector space does not hold for minimal resolving sets of quasi flower snarks, generalized prism and generalized antiprism.
\end{abstract}

Keywords: Metric dimension, basis, resolving set, exchange property, quasi flower snark.

2010 Mathematics Subject Classification: $05 \mathrm{C} 12$

\section{Introduction and preliminary results}

Metric dimension is a parameter that has appeared in various applications of graph theory, diverse as, pharmaceutical chemistry [7], robot navigation [18], combinatorial optimization [21] and sonar and coast guard Loran [22], to name a few. Metric dimension is a generalization of affine dimension to arbitrary metric spaces (provided a resolving set exists).

In a connected graph $G$, the distance $d(u, v)$ between two vertices $u, v \in V(G)$ is the length of a shortest path between them. Let $W=\left\{w_{1}, w_{2}, \ldots, w_{k}\right\}$ be an ordered set of vertices of $G$ and let $v$ be a vertex of $G$. The representation $r(v \mid W)$ of $v$ with respect to $W$ is the $k$-tuple $\left(d\left(v, w_{1}\right), d\left(v, w_{2}\right), d\left(v, w_{3}\right), \ldots, d\left(v, w_{k}\right)\right) . W$ is called a resolving set [7] or locating set [22] if every vertex of $G$ is uniquely identified by its distances from the vertices of $W$, or equivalently, if distinct vertices of $G$ have distinct representations with respect to $W$. A resolving set of minimum cardinality is called a basis for $G$ and this cardinality is the metric dimension or location number of $G$, denoted by $\beta(G)$ [5]. The concepts of resolving set and metric basis have previously appeared in the literature (see [1-7, 9-26]).

For a given ordered set of vertices $W=\left\{w_{1}, w_{2}, \ldots, w_{k}\right\}$ of a graph $G$, the $i$ th component of $r(v \mid W)$ is 0 if and only if $v=w_{i}$. Thus, to show that $W$ is a resolving set it suffices to verify that $r(x \mid W) \neq r(y \mid W)$ for each pair of distinct vertices $x, y \in V(G) \backslash W$.

A useful property in finding $\beta(G)$ is the following lemma:

Lemma 1.1.[23] Let $W$ be a resolving set for a connected graph $G$ and $u, v \in V(G)$. If $d(u, w)=d(v, w)$ for all vertices $w \in V(G) \backslash\{u, v\}$, then $\{u, v\} \cap W \neq \emptyset$.

Let $\mathscr{F}$ be a family of connected graphs $G_{n}: \mathscr{F}=\left(G_{n}\right)_{n \geq 1}$ depending on $n$ as follows: the order $|V(G)|=\varphi(n)$ and $\lim _{n \rightarrow \infty} \varphi(n)=\infty$. If there exists a constant $C>0$ such that $\beta\left(G_{n}\right) \leq C$ for every $n \geq 1$, then we shall say that $\mathscr{F}$ has bounded metric dimension; otherwise $\mathscr{F}$ has unbounded metric dimension.

If all graphs in $\mathscr{F}$ have the same metric dimension (which does not depend on $n$ ), $\mathscr{F}$ is called a family with

\footnotetext{
*Corresponding author e-mail: imrandhab@gmail.com
} 
constant metric dimension [16]. A connected graph $G$ has $\beta(G)=1$ if and only if $G$ is a path [7]; cycles $C_{n}$ have metric dimension 2 for every $n \geq 3$. Also generalized Petersen graphs $P(n, 2)$, antiprisms $A_{n}$ and circulant graphs $C_{n}^{2}$ are families of graphs with constant metric dimension [16]. Some classes of regular graphs with constant metric dimension have been studied in $[1,3,10]$ recently while metric dimension of some classes of convex polytopes has been determined in [11] and [13]. The metric dimension of graphs with pendent edges has been investigated in [15]. The metric dimension of necklace graphs $N e_{n}$ has been calculated by using the idea of resolving pairs in [25].

Other families of graphs have unbounded metric dimension: if $W_{n}$ denotes a wheel with $n$ spokes and $J_{2 n}$ the graph deduced from the wheel $W_{2 n}$ by alternately deleting $n$ spokes, then $\beta\left(W_{n}\right)=\left\lfloor\frac{2 n+2}{5}\right\rfloor$ for every $n \geq 7$ [5] and $\beta\left(J_{2 n}\right)=\left\lfloor\frac{2 n}{3}\right\rfloor$ [26] for every $n \geq 4$.

The cartesian product of $G$ and $H$ is a graph, denoted by $G \square H$, whose vertex set is $V(G) \times V(H)$. Two vertices $(g, h)$ and $\left(g^{\prime}, h^{\prime}\right)$ are adjacent precisely if $g=g^{\prime}$ and $h h^{\prime} \in E(H)$, or $g g^{\prime} \in E(G)$ and $h=h^{\prime}$. Thus, $V(G \square H)=\{(g, h) \mid g \in V(G)$ and $h \in V(H)\}$ and $E(G \square H)=\left\{(g, h)\left(g^{\prime}, h^{\prime}\right) \mid g=g^{\prime}, h h^{\prime} \in E(H)\right.$ or $\left.g g^{\prime} \in E(G), h=h^{\prime}\right\}$.

An example of a family which has bounded metric dimension is the family of prisms denoted by $D_{n} \cong P_{m} \square C_{n}$. In [6] it was proved that

$$
\beta\left(P_{m} \square C_{n}\right)=\left\{\begin{array}{l}
2, \text { if } n \text { is odd; } \\
3, \text { otherwise. }
\end{array}\right.
$$

Since prisms $D_{n}$ are the cubic plane graphs obtained by the cartesian product of path $P_{2}$ with a cycle $C_{n}$, so prisms constitute a family of cubic graphs with bounded metric dimension. Also generalized Petersen graphs $P(n, 3)$ have bounded metric dimension [12]. The metric dimension of the lexicographic product of graphs has been studied in [20].

The graphs having metric dimension 1 are characterized in the following theorem.

Theorem 1.1.[7] The metric dimension of a graph $G$ is 1 if and only if $G \cong P_{n}$, where $P_{n}$ denotes a path of length $n-1$ or $G$ is one-way infinite path.

The next theorem gives a nice property of the graphs with metric dimension 2 .

Theorem 1.2. [17] Let $G$ be a graph with metric dimension 2 and let $\left\{v_{1}, v_{2}\right\} \subseteq V(G)$ be a metric basis in $G$, then the degree of both $v_{1}$ and $v_{2}$ is at most 3 .

Note that the problem of determining whether $\beta(G)<k$ is an $N P$-complete problem [8].

In this paper, we study the metric dimension of some graphs that are rotationally-symmetric namely quasi flower snarks, generalized antiprism and cartesian product of square cycle with a path. We prove that these classes of graphs have constant or bounded metric dimension. It is natural to ask for characterization of graphs classes with respect to the nature of their metric dimension. It is also shown that the exchange property of the bases in a vector space does not hold for minimal resolving sets of generalized prism, quasi flower snarks and generalized antiprism.

\section{The metric dimension of quasi flower snarks}

The quasi flower snark denoted by $G_{n}$ is a nontrivial simple connected cubic graph, where $V\left(G_{n}\right)=\left\{a_{i}, b_{i}, c_{i}, d_{i}: 0 \leq i \leq n-1\right\}$ and $E\left(G_{n}\right) \quad\left\{\quad\left\{a_{i} a_{i+1}, b_{i} b_{i+1}, c_{i} c_{i+1}, a_{i} d_{i}\right.\right.$, $\left.b_{i} d_{i}, c_{i} d_{i}: 0 \leq i \leq n-1\right\}$, the indices are taken modulo $n$. The quasi flower snarks $G_{10}$ and $G_{9}$ are depicted in Fig. 1. The metric dimension of flower snarks has been studied

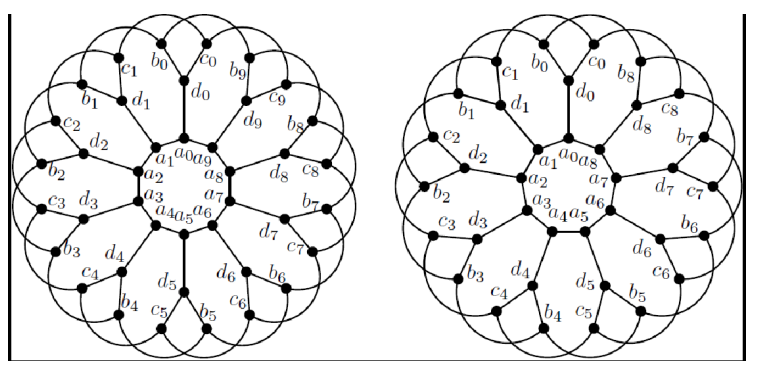

Fig. 1: Quasi flower snarks $G_{10}$ and $G_{9}$

in [10] where it was proved that flower snakrs constitute a family of cubic graphs with constant metric dimension 3 . In the next theorem, we extend this study to the metric dimension of quasi flower snarks.

Theorem 2.1. Let $G_{n}$ be the quasi flower snark. Then for every positive integer $n \geq 4$ we have

$$
\beta\left(G_{n}\right)= \begin{cases}3, & \text { if } \mathrm{n} \text { is odd } \\ \leq 4, & \text { otherwise }\end{cases}
$$

Proof. We consider the following cases.

Case (1). When $n \equiv 0(\bmod 2)$. Then we can write as $n=2 k$, where $k \geq 2$. Suppose $W=\left\{b_{0}, c_{0}, d_{n-1}\right\}$ is a resolving set. For this, we give the representations of $V\left(G_{n}\right) \backslash W$.

$r\left(a_{i} \mid W\right)$
$\left\{\begin{array}{lr}(i+2, i+2, i+2), & 0 \leq i \leq k-1 \\ (2 k-i+2,2 k-i+2,2 k-i), & k \leq i \leq 2 k-1 .\end{array}\right.$

$r\left(b_{i} \mid W\right)=\left\{\begin{array}{lr}(i, i+2, i+2), & 0 \leq i \leq k-1 \\ (2 k-i, 2 k-i+2,2 k-i), & k \leq i \leq 2 k-1 .\end{array}\right.$ 
$r\left(c_{i} \mid W\right)=\left\{\begin{array}{lr}(i+2, i, i+2), & 0 \leq i \leq k-1 \\ (2 k-i+2,2 k-i, 2 k-i), & k \leq i \leq 2 k-1\end{array}\right.$

and

$r\left(d_{i} \mid W\right)$

$\left\{\begin{array}{rr}(i+1, i+1, i+3), & 0 \leq i \leq k-1 ; \\ (2 k-i+1,2 k-i+1,2 k-i+1), & k \leq i \leq 2 k-2 .\end{array}\right.$

It can be seen that for $1 \leq i \leq k-1, r\left(a_{i} \mid W\right)=r\left(d_{2 k-i-1} \mid\right.$ $W)=(i+2, i+2, i+2)$. In order to have distinct representations, we add the vertex $a_{0}$ to $W$. Then for $1 \leq i \leq k-1$, we have $d\left(a_{0}, a_{i}\right)=i$ and $d\left(a_{0}, d_{2 k-i-1}\right)=i+2$. Then we have $W^{\prime}=W \cup\left\{a_{0}\right\}$, that resolves vertices of $G_{n}$ when $n \equiv 0(\bmod 2)$. Thus $\beta\left(G_{n}\right) \leq 4$ when $n \equiv 0(\bmod 2)$.

Case (2). When $n \equiv 1(\bmod 2)$. Then we have $n=2 k+1 ; k \geq 2$. We will prove this case by double inequality. First, we show that $W=\left\{b_{0}, c_{0}, c_{k}\right\}$ is a resolving set for $G_{n}$. For this, first we give representations of $V\left(G_{n}\right) \backslash W$. Then

$$
\begin{aligned}
& r\left(a_{i} \mid W\right) \\
& \left\{\begin{array}{rr}
(i+2, i+2, k-i+2), & 0 \leq i \leq k \\
(k+2, k+2,3), & i=k+1 \\
(2 k-i+3,2 k-i+3, i-k+2), & \\
k+2 \leq i \leq 2 k
\end{array}\right.
\end{aligned}
$$

$r\left(b_{i} \mid W\right)$

$\left\{\begin{array}{rr}(i, i+2, k-i+2), & 0 \leq i \leq k \\ (k, k+2,3), & i=k+1 \\ (2 k-i+1,2 k-i+3, i-k+2), & \\ k+2 \leq i \leq 2 k .\end{array}\right.$

$r\left(c_{i} \mid W\right)=\left\{\begin{array}{lr}(i+2, i, k-i), & 0 \leq i \leq k \\ (k+2, k, 1), & i=k+1 \\ (2 k-i+3,2 k-i+1, i-k), k+2 \leq i \leq 2 k\end{array}\right.$

and

$r\left(d_{i} \mid W\right)$

$\left\{\begin{array}{rr}(i+1, i+1, k-i+1), & 0 \leq i \leq k ; \\ (k+1, k+1,2), & i=k+1 ; \\ (2 k-i+2,2 k-i+2, i-k+1), & \\ k+2 \leq i \leq 2 k .\end{array}\right.$

It can be seen that all vertices in $G_{n}$ have distinct representations implying that $\beta\left(G_{n}\right) \leq 3$ when $n \equiv 1$ $(\bmod 2)$.

On the other hand, we show that $\beta\left(G_{n}\right) \geq 3$. Contrarily suppose that $\beta\left(G_{n}\right)=2$, then there are discussed the following possibilities.

- If both vertices belong to the set $\left\{a_{0}, a_{1}, \cdots, a_{n-1}\right\}$, then we choose the resolving set $W=\left\{a_{p}, a_{q}\right\}$, where $0 \leq p<q \leq 2 k$. However, then we get

$r\left(b_{p} \mid W\right)=r\left(c_{p} \mid W\right)$

$$
=\left\{\begin{array}{lr}
(2, q-p+2), & 1 \leq q-p \leq k ; \\
(2,2 k-q+p+3), k+1 \leq q-p \leq 2 k
\end{array}\right.
$$

a contradiction.

- If both vertices belong to the set $\left\{d_{0}, d_{1}, \cdots, d_{n-1}\right\}$. We suppose, resolving set is $W=\left\{d_{p}, d_{q}\right\}$, where $0 \leq p<q \leq 2 k$. However, then we have

$$
\begin{aligned}
r\left(b_{p} \mid W\right) & =r\left(c_{p} \mid W\right) \\
& =\left\{\begin{array}{lr}
(1, q-p+1), & 1 \leq q-p \leq k ; \\
(1,2 k-q+p+2), k+1 \leq q-p \leq 2 k
\end{array}\right.
\end{aligned}
$$

a contradiction.

- If both vertices belong to either $\left\{b_{0}, b_{1}, \cdots, b_{n-1}\right\}$ or $\left\{c_{0}, c_{1}, \cdots, c_{n-1}\right\}$. Without loss of generality we assume that both vertices belong to the set $\left\{b_{0}, b_{1}, \cdots, b_{n-1}\right\}$. Then we may choose the resolving set $W=\left\{b_{p}, b_{q}\right\}$, where $0 \leq p<q \leq 2 k$. Then

$$
\begin{aligned}
r\left(a_{p} \mid W\right) & =r\left(c_{p} \mid W\right) \\
& =\left\{\begin{array}{lr}
(2, q-p+2), & 1 \leq q-p \leq k ; \\
(2,2 k-q+p+3), & k+1 \leq q-p \leq 2 k
\end{array}\right.
\end{aligned}
$$

a contradiction

- If one vertex belongs to the set $\left\{b_{0}, b_{1}, \cdots, b_{n-1}\right\}$ and other one is in the set $\left\{c_{0}, c_{1}, \cdots, c_{n-1}\right\}$. Without loss of generality we suppose, resolving set is $W=\left\{b_{p}, c_{q}\right\}$, where $0 \leq p \leq q \leq 2 k$. However, then

$r\left(a_{p+1} \mid W\right)=r\left(a_{p-1} \mid W\right)=(3,3)$ when $p=q$;

$r\left(a_{p} \mid W\right)=r\left(d_{p-1} \mid W\right)=(2, q-p+2) \quad$ when $1 \leq q-p \leq k-1$

$r\left(c_{p+1} \mid W\right)=r\left(d_{p+2} \mid W\right)=(3, q-p-1) \quad$ when $q-p=k, k+1$

$r\left(a_{p} \mid W\right)=r\left(d_{p+1} \mid W\right)=(2,2 k-q+p+3)$ when $k+2 \leq q-p \leq 2 k$.

The indices are taken modulo $2 k+1$. We get a contradiction in each subcase.

- If one vertex belongs to the set $\left\{a_{0}, a_{1}, \cdots, a_{n-1}\right\}$ and the other vertex belongs to the set $\left\{d_{0}, d_{1}, \cdots, d_{n-1}\right\}$. Without loss of generality, we can take $W=\left\{d_{p}, a_{q}\right\}$, where $0 \leq p \leq q \leq 2 k$. However, we have

$$
\begin{aligned}
r\left(b_{p} \mid W\right) & =r\left(c_{p} \mid W\right) \\
& =\left\{\begin{array}{lr}
(1, q-p+2), & 0 \leq q-p \leq k ; \\
(1,2 k-q+p+3), & k+1 \leq q-p \leq 2 k
\end{array}\right.
\end{aligned}
$$

a contradiction.

- If one vertex belongs to the set $\left\{a_{0}, a_{1}, \cdots, a_{n-1}\right\}$ and other vertex belongs to either $\left\{b_{0}, b_{1}, \cdots, b_{n-1}\right\}$ or $\left\{c_{0}, c_{1}, \cdots, c_{n-1}\right\}$. Without loss of generality we suppose, the second vertex belongs to the set $\left\{b_{0}, \cdots, b_{n-1}\right\}$. Then we can choose $W=\left\{a_{p}, b_{q}\right\}$, where $0 \leq p \leq q \leq 2 k$. But then we get

$r\left(a_{p-1} \mid W\right)=r\left(a_{p+1} \mid W\right)=(1,3)$ when $p=q$;

$r\left(a_{p+1} \mid W\right)=r\left(d_{p} \mid W\right)=(1, q-p+1) \quad$ when $0 \leq q-p \leq k$

$r\left(\bar{a}_{p-1} \mid W\right)=r\left(d_{p} \mid W\right)=(1,2 k-q+p+2)$ when $k+1 \leq q-p \leq 2 k$.

The indices are taken modulo $2 k+1$, a contradiction.

- If one vertex belongs to the set $\left\{d_{0}, d_{1}, \cdots, d_{n-1}\right\}$ and other vertex belongs to either $\left\{b_{0}, b_{1}, \cdots, b_{n-1}\right\}$ or 
$\left\{c_{0}, c_{1}, \cdots, c_{n-1}\right\}$. Without loss of generality we suppose, the second vertex belongs to the set $\left\{b_{0}, b_{1}, \cdots, b_{n-1}\right\}$. We may suppose that the resolving set is $W=\left\{b_{p}, d_{q}\right\}$, where $0 \leq p \leq q \leq 2 k$. But then

$$
\begin{aligned}
r\left(a_{p} \mid W\right) & =r\left(c_{p} \mid W\right) \\
& =\left\{\begin{array}{lr}
(2, q-p+1), & 0 \leq q-p \leq k \\
(2,2 k-q+p+2), & k+1 \leq q-p \leq 2 k
\end{array}\right.
\end{aligned}
$$

We get a contradiction.

This yields that $\beta\left(G_{n}\right) \geq 3$. So from above, we conclude that there is no resolving set with two vertices of $G_{n}$. Hence $\beta\left(G_{n}\right)=3$ when $n \equiv 1(\bmod 2)$.

\section{Metric dimension of cartesian product of square cycle and path}

The metric dimension of cartesian product of cycle and path has been investigated in [6]. In this section, we extend this study to the cartesian product of square cycle and path and prove that the cartesian product of square cycle and path have metric dimension equal to 3 when $n \equiv 0,2,3(\bmod 4)$ and at most 4 otherwise. For $t \geq 2$, we have $V\left(C_{n}^{2} \square P_{t}\right)=\left\{u_{s}^{i}: 1 \leq s \leq t, 1 \leq i \leq n\right\}$. In the next theorem, we determine the metric dimension of cartesian product of square cycle and path. Note that the choice of appropriate basis vertices (also called landmarks) is core of the problem.

Theorem 3.1. For every positive integer $n \geq 5$,

$$
\beta\left(C_{n}^{2} \square P_{t}\right)=\left\{\begin{array}{l}
3, \quad \text { when } n \equiv 0,2,3(\bmod 4) ; \\
\leq 4, \text { otherwise. }
\end{array}\right.
$$

Proof. We prove this theorem by double inequality. First we prove that

$$
\beta\left(C_{n}^{2} \square P_{t}\right) \leq\left\{\begin{array}{l}
3, \text { when } n \equiv 0,2,3(\bmod 4) ; \\
4, \text { otherwise }
\end{array}\right.
$$

by showing that $W=\left\{u_{1}^{1}, u_{1}^{3}, u_{t}^{2}\right\}$ resolves all vertices of $C_{n}^{2} \square P_{t}$ when $n \equiv 0,2,3(\bmod 4)$. For this, we give representations of $V\left(C_{n}^{2} \square P_{t}\right) \backslash W$ in each case.

Case $(1)$. When $n \equiv 0(\bmod 4)$. Then we can write as $n=4 k$, where $k \geq 2$. For $s=1$,

$$
\begin{aligned}
& r\left(u_{s}^{2 i} \mid W\right) \\
& \left\{\begin{array}{lr}
(1,1, t-1), & i=1 ; \\
(i, i-1, t+i-2), & 2 \leq i \leq k ; \\
(k, k, t+k-1), & i=k+1 ; \\
(2 k-i+1,2 k-i+2,2 k+t-i), & k+2 \leq i \leq 2 k .
\end{array}\right.
\end{aligned}
$$$$
\begin{aligned}
& r\left(u_{s}^{2 i+1} \mid W\right) \\
& \left\{\begin{array}{r}
(i, i-1, t+i-1), \quad 2 \leq i \leq k ; \\
(2 k-i, 2 k-i+1,2 k+t-i), \\
k+1 \leq i \leq 2 k-1 .
\end{array}\right.
\end{aligned}
$$

For $s=t$,

$$
\begin{aligned}
& r\left(u_{s}^{2 i} \mid W\right) \\
& \left\{\begin{array}{lr}
(t+i-1, t+i-2, i-1), & 2 \leq i \leq k \\
(k+t-1, k+t-1, k), & i=k+1 \\
(2 k+t-i, 2 k+t-i+1,2 k-i+1) & k+2 \leq i \leq 2 k
\end{array}\right.
\end{aligned}
$$$$
r\left(u_{s}^{2 i+1} \mid W\right)
$$$$
\left\{\begin{array}{rr}
(t-1, t, 1), & i=0 ; \\
(t+i-1, t+i-2, i), & 1 \leq i \leq k ; \\
(2 k+t-i-1,2 k+t-i, 2 k-i+1), & k+1 \leq i \leq 2 k-1
\end{array}\right.
$$

and for $2 \leq s \leq t-1$, we have

$r\left(u_{s}^{2 i} \mid W\right)$
$\left\{\begin{array}{rr}(s, s, t-s), & i=1 ; \\ (s+i-1, s+i-2, t-s+i-1), & 2 \leq i \leq k \\ (k+s-1, k+s-1, k+t-s), & i=k+1 \\ (2 k+s-i, 2 k+s-i+1,2 k+t-s-i+1), & k+2 \leq i \leq 2 k .\end{array}\right.$

$$
\begin{aligned}
& r\left(u_{s}^{2 i+1} \mid W\right) \\
& \left\{\begin{array}{l}
(s-1, s, t-s+1), \quad i=0 ; \\
(s+i-1, s+i-2, t-s+i), \quad 1 \leq i \leq k ; \\
(2 k+s-i-1,2 k+s-i, 2 k+t \\
-s-i+1), \quad k+1 \leq i \leq 2 k-1 .
\end{array}\right.
\end{aligned}
$$

Since all the vertices have distinct representations with respect to $W$. This yields $\beta\left(C_{n}^{2} \square P_{t}\right) \leq 3$ when $n \equiv 0$ $(\bmod 4)$.

Case $(2)$. When $n \equiv 2(\bmod 4)$. Then we can write as $n=4 k+2$, where $k \geq 1$. For $s=1$,

$$
\begin{aligned}
& r\left(u_{s}^{2 i} \mid W\right) \\
& \left\{\begin{array}{rr}
(1,1, t-1), & i=1 ; \\
(i, i-1, t+i-2), & 2 \leq i \leq k+1 ; \\
(2 k-i+2,2 k-i+3,2 k+t-i+1), & k+2 \leq i \leq 2 k+1 .
\end{array}\right.
\end{aligned}
$$$$
\begin{aligned}
& r\left(u_{s}^{2 i+1} \mid W\right) \\
& \left\{\begin{array}{rr}
(i, i-1, t+i-1), & 2 \leq i \leq k \\
(k, k, t+k), & i=k+1 \\
(2 k-i+1,2 k-i+2,2 k+t-i+1), & k+2 \leq i \leq 2 k .
\end{array}\right.
\end{aligned}
$$

For $s=t$,

$$
\begin{aligned}
& r\left(u_{s}^{2 i} \mid W\right) \\
& \left\{\begin{array}{r}
(t+i-1, t+i-2, i-1), \quad 2 \leq i \leq k+1 \\
(2 k+t-i+1,2 k+t-i+2,2 k-i+2) \\
k+2 \leq i \leq 2 k+1 .
\end{array}\right.
\end{aligned}
$$

$$
\begin{aligned}
& r\left(u_{s}^{2 i+1} \mid W\right) \\
& \left\{\begin{array}{rr}
(t-1, t, 1), & i=0 \\
(t+i-1, t+i-2, i), & 1 \leq i \leq k \\
(k+t-1, k+t-1, k+1), & i=k+1 \\
(2 k+t-i, 2 k+t-i+1,2 k-i+2), & k+2 \leq i \leq 2 k
\end{array}\right.
\end{aligned}
$$


and for $2 \leq s \leq t-1$, we have

$r\left(u_{s}^{2 i} \mid W\right)$
$\left\{\begin{array}{l}(s, s, t-s), \\ (s+i-1, s+i-2, t-s+i-1), \\ 2 \leq i \leq k+1 ; \\ (2 k+s-i+1,2 k+s-i+2,2 k+t \\ -s-i+2), \quad k+2 \leq i \leq 2 k+1 .\end{array}\right.$

$r\left(u_{s}^{2 i+1} \mid W\right)$
$\left\{\begin{array}{rr}(s-1, s, t-s+1), & i=0 \\ (s+i-1, s+i-2, t-s+i), & 1 \leq i \leq k \\ (k+s-1, k+s-1, k+t-s+1) & i=k+1 \\ (2 k+s-i, 2 k+s-i+1,2 k+t & k+2 \leq i \leq 2 k \\ -s-i+2), & k+2\end{array}\right.$

It can be verified that all vertices have distinct representations with respect to $W$. This yields $\beta\left(C_{n}^{2} \square P_{t}\right) \leq 3$ when $n \equiv 2(\bmod 4)$.

Case $(3)$. When $n \equiv 3(\bmod 4)$. Then we can write as $n=4 k+3$, where $k \geq 1$. For $s=1$,

$r\left(u_{s}^{2 i} \mid W\right)$

$\left\{\begin{array}{rr}(1,1, t-1), & i=1 ; \\ (i, i-1, t+i-2), & 2 \leq i \leq k+1 ; \\ (2 k-i+2,2 k-i+3,2 k+t-i+2), & k+2 \leq i \leq 2 k+1 .\end{array}\right.$

$r\left(u_{s}^{2 i+1} \mid W\right)$

$\left\{\begin{array}{rr}(i, i-1, t+i-1), & 2 \leq i \leq k+1 ; \\ (2 k-i+2,2 k-i+3,2 k+t-i+1), \\ k+2 \leq i \leq 2 k+1 .\end{array}\right.$

For $s=t$,

$r\left(u_{s}^{2 i} \mid W\right)$

$\left\{\begin{array}{r}(t+i-1, t+i-2, i-1), \quad 2 \leq i \leq k+1 \\ (2 k+t-i+1,2 k+t-i+2,2 k-i+3) \\ k+2 \leq i \leq 2 k+1 .\end{array}\right.$

$r\left(u_{s}^{2 i+1} \mid W\right)$

$\left\{\begin{array}{lr}(t-1, t, 1), & i=0 ; \\ (t+i-1, t+i-2, i), & 1 \leq i \leq k ; \\ (k+t, k+t-1, k+1), & i=k+1 ; \\ (2 k+t-i+1,2 k+t-i+2,2 k-i+2), & k+2 \leq i \leq 2 k+1\end{array}\right.$

and for $2 \leq s \leq t-1$, we have

$$
\begin{aligned}
& r\left(u_{s}^{2 i} \mid W\right) \\
& \left\{\begin{array}{c}
(s, s, t-s), \\
(s+i-1, s+i-2, t-s+i-1) \\
2 \leq i \leq k+1 \\
(2 k+s-i+1,2 k+s-i+2,2 k+t \\
-s-i+3), \quad k+2 \leq i \leq 2 k+1 .
\end{array}\right.
\end{aligned}
$$

$$
=
$$

$$
\begin{aligned}
& r\left(u_{s}^{2 i+1} \mid W\right) \\
& \left\{\begin{array}{l}
(s-1, s, t-s+1), \quad i=0 \\
(s+i-1, s+i-2, t-s+i), \quad 1 \leq i \leq k \\
(k+s, k+s-1, k+t-s+1), \quad i=k+1 \\
(2 k+s-i+1,2 k+s-i+2,2 k+t \\
-s-i+2), \quad k+2 \leq i \leq 2 k+1
\end{array}\right.
\end{aligned}
$$

Again, in this case all the vertices have distinct representations with respect to $W$. This yields $\beta\left(C_{n}^{2} \square P_{t}\right) \leq 3$ when $n \equiv 3(\bmod 4)$.

Case(4). When $n \equiv 1(\bmod 4)$. Then we can write as $n=4 k+1$, where $k \geq 1$. For $s=1$,

$$
\begin{aligned}
& r\left(u_{s}^{2 i} \mid W\right) \\
& \left\{\begin{array}{lr}
(1,1, t-1), & i=1 ; \\
(i, i-1, t+i-2), & 2 \leq i \leq k ; \\
(k, k, k+t-1), & i=k+1 ; \\
(2 k-i+1,2 k-i+2,2 k+t-i+1), & k+2 \leq i \leq 2 k .
\end{array}\right.
\end{aligned}
$$$$
\begin{aligned}
& r\left(u_{s}^{2 i+1} \mid W\right) \\
& \left\{\begin{array}{rr}
(i, i-1, t+i-1), & 2 \leq i \leq k ; \\
(k, k, k+t-1), & i=k+1 ; \\
(2 k-i+1,2 k-i+2,2 k+t-i), & k+2 \leq i \leq 2 k .
\end{array}\right.
\end{aligned}
$$

For $s=t$,

$$
\begin{aligned}
& r\left(u_{s}^{2 i} \mid W\right) \\
& \left\{\begin{array}{lr}
(t+i-1, t+i-2, i-1), & 2 \leq i \leq k ; \\
(k+t-1, k+t-1, k), & i=k+1 ; \\
(2 k+t-i, 2 k+t-i+1,2 k-i+2), & k+2 \leq i \leq 2 k .
\end{array}\right.
\end{aligned}
$$

$$
\begin{aligned}
& r\left(u_{s}^{2 i+1} \mid W\right) \\
& \left\{\begin{array}{lr}
(t-1, t, 1), & i=0 \\
(t+i-1, t+i-2, i), & 1 \leq i \leq k \\
(k+t-1, k+t-1, k), & i=k+1 \\
(2 k+t-i, 2 k+t-i+1,2 k-i+1), & k+2 \leq i \leq 2 k
\end{array}\right.
\end{aligned}
$$

and for $2 \leq s \leq t-1$, we have

$r\left(u_{s}^{2 i} \mid W\right)$

$\left\{\begin{array}{rr}(s, s, t-s), & i=1 ; \\ (s+i-1, s+i-2, t-s+i-1), & 2 \leq i \leq k ; \\ (k+s-1, k+s-1, k+t-s), \quad i=k+1 & i \leq \\ (2 k+s-i, 2 k+s-i+1,2 k+t-s-i+2) & k+2 \leq i \leq 2 k .\end{array}\right.$

$r\left(u_{s}^{2 i+1} \mid W\right)$
$\left\{\begin{array}{lr}(s-1, s, t-s+1), & i=0 \\ (s+i-1, s+i-2, t-s+i), \quad 1 \leq i \leq k \\ (k+s-1, k+s-1, k+t-s), \quad i=k+1 \\ (2 k+s-i, 2 k+s-i+1,2 k+t \\ -s-i+1), \quad k+2 \leq i \leq 2 k\end{array}\right.$

It can be seen that for $1 \leq s \leq t$, we have $r\left(u_{s}^{2 k+2} \mid W\right)=$ 
$r\left(u_{s}^{2 k+3} \mid W\right)=(k+s-1, k+s-1, k+t-s)$. If we add the vertex $u_{t}^{4}$ to $W$. Then $d\left(u_{t}^{4}, u_{s}^{2 k+2}\right)=k+t-s-1$ and $d\left(u_{t}^{4}, u_{s}^{2 k+3}\right)=k+t-s$, where $1 \leq s \leq t$. Thus $W^{\prime}=W \cup\left\{u_{t}^{4}\right\}$ resolves vertices of $C_{n}^{2} \square P_{t}$. This yields $\beta\left(C_{n}^{2} \square P_{t}\right) \leq 4$ when $n \equiv 1(\bmod 4)$.

Conversely, we show that $\beta\left(C_{n}^{2} \square P_{t}\right) \geq 3$ when $n \equiv 0,2,3(\bmod 4)$. Suppose contrarily $\beta\left(C_{n}^{2} \square P_{t}\right)=2$. Then by Theorem 1.2 we get a contradiction. Thus $\beta\left(C_{n}^{2} \square P_{t}\right) \geq 3$ when $n \equiv 0,2,3(\bmod 4)$. Hence $\beta\left(C_{n}^{2} \square P_{t}\right)=3$ for $n \equiv 0,2,3(\bmod 4)$, which completes the proof.

\section{Metric dimension of generalized antiprism}

A generalized antiprism $A_{n}^{m}$ can be obtained by completing the generalized prism $C_{n} \square P_{m}$ by edges $\left\{v_{i, l+1} v_{i+1, l}: 0 \leq i \leq n-2,1 \leq l \leq\right.$ $m-1\} \cup\left\{v_{n-1, l+1} \bar{v}_{0, l}: 1 \leq l \leq m-1\right\}$. Let $V\left(A_{n}^{m}\right) \quad=\quad V\left(C_{n} \square P_{m}\right) \quad$ and $E\left(A_{n}^{m}\right)=E\left(C_{n} \square P_{m}\right) \cup\left\{v_{i, l+1} v_{i+1, l}: 0 \leq i \leq n-2,1 \leq l \leq\right.$ $m-1\} \cup\left\{v_{n-1, l+1} v_{0, l}: 1 \leq l \leq m-1\right\}$ be the edge set of $A_{n}^{m}$, where $i$ is taken modulo $n$. The metric dimension of antiprism denoted by $A_{n}^{2}$ has been determined in [16]. In the next theorem, we determine the metric dimension of generalized antiprism.

Theorem 4.1. Let $A_{n}^{m}$ be the generalized antiprism. Then for every positive integer $n \geq 6$, we have

$$
\beta\left(A_{n}^{m}\right)= \begin{cases}3, & \text { if } 2 \leq m \leq 5 \\ \geq 4, & \text { if } m \geq 6\end{cases}
$$

Proof. We denote $V\left(A_{n}^{m}\right)=\left\{v_{i, l}: 0 \leq i \leq n-1,1 \leq l \leq\right.$ $m\}$. When $m=2, A_{n}^{2} \cong A_{n}$ (antiprism), and it was proved in [16] that $\beta\left(A_{n}\right)=3$. Now, we prove that $\beta\left(A_{n}^{m}\right)=3$ for $3 \leq m \leq 5$ and $n \geq 6$ by double inequality. We show that $W=\left\{v_{2,1}, v_{0,\left\lfloor\frac{m-1}{2}\right\rfloor}, v_{0, m}\right\}$ resolves all vertices of $A_{n}^{m}$ by giving the representations of $V\left(A_{n}^{m}\right) \backslash W$.

Case (1). When $n=2 k ; k \geq 3$. First, we will give the representations of all vertices of $V\left(A_{n}^{m}\right) \backslash W$ when $3 \leq m \leq 4$. For $l=1$, we have $r\left(v_{1, l} \mid W\right)=(1,1, m-1)$, and

$$
\begin{aligned}
& r\left(v_{i, l} \mid W\right) \\
& \left\{\begin{array}{rr}
(i-2, i, i), & 3 \leq i \leq k ; \\
(k-1, k-1, k+1), & i=k+1 \\
(2 k-i+2,2 k-i, 2 k+m-i-1), & \\
k+2 \leq i \leq 2 k-1 .
\end{array}\right.
\end{aligned}
$$

For $l=m, \quad r\left(v_{1, l} \mid W\right)=(l-1, l, 1)$, $r\left(v_{2 k-2, l} \mid W\right)=(4, l-1,2), r\left(v_{2 k-1, l} \mid W\right)=(3, l-1,1)$ and

$$
\begin{aligned}
& r\left(v_{i, l} \mid W\right) \\
& \left\{\begin{array}{lr}
(i+l-3, i+l-1, i), & 2 \leq i \leq k-2 \\
(i+l-3,2 k-i, i), & i=k-1, k \\
(2 k-i+2,2 k-i, 2 k-i), & \\
k+1 \leq i \leq 2 k-3
\end{array}\right.
\end{aligned}
$$

and for $2 \leq l \leq m-1$, we have $r\left(v_{0, l} \mid W\right)=(2, l-1, m-l), r\left(v_{1, l} \mid W\right)=(l-1, l, m-l)$, $r\left(v_{2 k-1, l} \mid W\right)=(3, l-1, m-l+1)$ and

$r\left(v_{i, l} \mid W\right)$

$\left\{\begin{array}{r}(i+l-3, i+l-1, i), \quad 2 \leq i \leq k-1 ; \\ (k+l-3, k, k), \quad i=k ; \\ (k+l-2, k-1, k+m-l-1), \quad i=k+1 ; \\ (2 k-i+2,2 k-i, 2 k+m-i-l), \\ k+2 \leq i \leq 2 k-2 .\end{array}\right.$

Now we will give the representations when $m=5$. For $l=1, \quad r\left(v_{0, l} \mid W\right)=(2,1,4), \quad r\left(v_{1, l} \mid W\right)=(1,1,4)$, $r\left(v_{3, l} \mid W\right)=(1,3,4)$ and

$r\left(v_{i, l} \mid W\right)=\left\{\begin{array}{lr}(i-2, i, i), & 4 \leq i \leq k ; \\ (k-1, k, k+1), & i=k+1 ; \\ (2 k-i+2,2 k-i+1,2 k-i+4), & k+2 \leq i \leq 2 k-1 .\end{array}\right.$

For $l=2, r\left(v_{1, l} \mid W\right)=(1,1,3), r\left(v_{2, l} \mid W\right)=(1,2,3)$ and

$r\left(v_{i, l} \mid W\right)=\left\{\begin{array}{rr}(i-1, i, i), & 3 \leq i \leq k ; \\ (k, k-1, k+1), & i=k+1 \\ (2 k-i+2,2 k-i, 2 k-i+3), & k+2 \leq i \leq 2 k-1 .\end{array}\right.$

For $l=m, r\left(v_{1, l} \mid W\right)=(4,4,1), r\left(v_{2 k-2, l} \mid W\right)=(4,3,2)$, $r\left(v_{2 k-1, l} \mid W\right)=(4,3,1)$ and

$r\left(v_{i, l} \mid W\right)=\left\{\begin{array}{lr}(i+2, i+3, i), & 2 \leq i \leq k-2 ; \\ (k+1, k+1, k-1), & i=k-1 ; \\ (2 k-i+2,2 k-i, 2 k-i), & k \leq i \leq 2 k-3\end{array}\right.$

and for $3 \leq l \leq m-1$, we have $r\left(v_{0, l} \mid W\right)=(l-1, l-2, m-l)$, $r\left(v_{1, l} \mid W\right)=(l-1, l-1, m-l)$, $r\left(v_{2 k-1, l} \mid W\right)=(3, l-2, m-l+1)$ and

$r\left(v_{i, l} \mid W\right)$

$\left\{\begin{array}{rr}(i+l-3, i+l-2, i), & 2 \leq i \leq k-1 ; \\ (k+l-3, k, k), & i=k ; \\ (2 k-i+2,2 k-i, 2 k+m-i-l), & k+1 \leq i \leq 2 k-2 .\end{array}\right.$

Case (2). When $n=2 k+1 ; k \geq 3$. For this, first we give the representations when $3 \leq m \leq 4$. For $l=1$, $r\left(v_{1, l} \mid W\right)=(1,1, m-l)$, and

$r\left(v_{i, l} \mid W\right)$

$\left\{\begin{array}{rr}(i-2, i, i), & 3 \leq i \leq k ; \\ (k-1, k, k+1), & i=k+1 ; \\ (k, k-1, k+m-2), & i=k+2 ; \\ (2 k-i+3,2 k-i+1,2 k+m-i), & k \leq i \leq 2 k .\end{array}\right.$

For $\quad l=m, \quad r\left(v_{1, l} \mid W\right)=(l-1, l, 1)$, $r\left(v_{2 k-1, l} \mid W\right)=(4, l-1,2), r\left(v_{2 k, l} \mid W\right)=(3, m-1,1)$ and

$r\left(v_{i, l} \mid W\right)$ 


$$
\left\{\begin{array}{rr}
(i+l-3, i+l-1, i), & 2 \leq i \leq k-1 ; \\
(k+l-3, k+1, k), & i=k ; \\
(k+l-2, k, k), & i=k+1 ; \\
(2 k-i+3,2 k-i+1,2 k-i+1), & k+2 \leq i \leq 2 k-2 .
\end{array}\right.
$$

For $2 \leq l \leq m-1, \quad r\left(v_{0, l} \mid W\right)=(2, l-1, m-l)$, $r\left(v_{1, l} \mid W\right)^{-}=(l \stackrel{-}{l} \quad 1, l, m-l)$, $r\left(v_{2 k, l} \mid W\right)=(3, l-1, m-l+1)$ and

$r\left(v_{i, l} \mid W\right)$

$\left\{\begin{array}{rr}(i+l-3, i+l-1, i), & 2 \leq i \leq k-1 ; \\ (i+l-3,2 k-i+1, i), & i=k, k+1 ; \\ (2 k-i+3,2 k-i+1,2 k+m-i-l+1), & k+2 \leq i \leq 2 k-1 .\end{array}\right.$

Now we will give the representations when $m=5$. For $l=1, \quad r\left(v_{0, l} \mid W\right)=(2,1,4), \quad r\left(v_{1, l} \mid W\right)=(1,1,4)$, $r\left(v_{3, l} \mid W\right)=(1,3,4)$ and

$$
\begin{aligned}
& r\left(v_{i, l} \mid W\right) \\
& \left\{\begin{array}{rr}
(i-2, i, i), & 4 \leq i \leq k+1 \\
(k, k, k+2), & i=k+2 ; \\
(2 k-i+3,2 k-i+2,2 k-i+5), & \\
k+3 & \leq i \leq 2 k .
\end{array}\right.
\end{aligned}
$$

For $l=2, r\left(v_{1, l} \mid W\right)=(1,1,3), r\left(v_{2, l} \mid W\right)=(1,2,3)$ and

$$
\begin{aligned}
& r\left(v_{i, l} \mid W\right) \\
& \left\{\begin{array}{rr}
(i-1, i, i), & 3 \leq i \leq k ; \\
(k, k, k+1), & i=k+1 ; \\
(2 k-i+3,2 k-i+1,2 k-i+4), & k+2 \leq i \leq 2 k .
\end{array}\right.
\end{aligned}
$$

For $l=m, r\left(v_{1, l} \mid W\right)=(4,4,1), r\left(v_{2 k-1, l} \mid W\right)=(4,3,2)$, $r\left(v_{2 k, l} \mid W\right)=(4,3,1)$ and

$$
r\left(v_{i, l} \mid W\right)=\left\{\begin{array}{lr}
(i+2, i+3, i), & 2 \leq i \leq k-1 ; \\
(k+2, k+1, k), & i=k ; \\
(2 k-i+3,2 k-i+1,2 k-i+1), & k+1 \leq i \leq 2 k-2
\end{array}\right.
$$

and for $3 \leq l \leq m-1$, we have $r\left(v_{0, l} \mid W\right)=(l-1, l-2, m-l)$, $r\left(v_{1, l} \mid W\right)=(l-1, l-1, m-l), r\left(v_{2 k, l} \mid W\right)=(3, l-2, m$ $-l+1)$ and

$$
\begin{aligned}
& r\left(v_{i, l} \mid W\right) \\
& \left\{\begin{array}{rr}
(i+l-3, i+l-2, i), & 2 \leq i \leq k-1 ; \\
(i+l-3,2 k-i+1, i), & i=k, k+1 ; \\
(2 k-i+3,2 k-i+1,2 k+m-i-l+1), & k+2 \leq i \leq 2 k-1 .
\end{array}\right.
\end{aligned}
$$$$
=
$$

It can be seen that all vertices of $A_{n}^{m}$ have distinct representations with respect to $W$. This shows that $\beta\left(A_{n}^{m}\right) \leq 3$ for $3 \leq m \leq 5$ and $n \geq 6$.

Conversely, suppose that $\beta\left(\bar{A}_{n}^{m}\right) \geq 3$, where $3 \leq m \leq 5$ and $n \geq 6$. Suppose on contrary that $\beta\left(A_{n}^{m}\right)=2$, but then by Theorem 1.2 we get a contradiction. Hence $\beta\left(A_{n}^{m}\right)=3$, when $2 \leq m \leq 5$ and $n \geq 6$.

\section{Exchange property for resolving sets in rotationally-symmetric graphs}

We have seen that a subset $W$ of vertices of a graph $G$ is a resolving set if every vertex in $G$ is uniquely determined by its distances to the vertices of $W$. Resolving sets behave like bases in a vector space in that each vertex in the graph can be uniquely identified relative to the vertices of these sets. But though resolving sets do share some of the properties of bases in a vector space, they do not always have the exchange property from linear algebra. Resolving sets are said to have the exchange property in $G$ if whenever $S$ and $R$ are minimal resolving sets for $G$ and $r \in R$, then there exists $s \in S$ so that $(S \backslash\{s\}) \cup\{r\}$ is a minimal resolving set [4].

If the exchange property holds for a graph $G$, then every minimal resolving set for $G$ has the same size and algorithmic methods for finding the metric dimension of $G$ are more feasible. Thus to show that the exchange property does not hold in a given graph, it is sufficient to show two minimal resolving sets of different size. However, since the converse is not true, knowing that the exchange property does not hold does not guarantee that there are minimal resolving sets of different size.

The following results concerning exchange property for resolving sets were deduced in [4].

Theorem 5.1.[4] The exchange property holds for resolving sets in trees.

Theorem 5.2. [4] For $n \geq 8$, resolving sets do not have the exchange property in wheels $W_{n}$.

It has been proved recently that exchange property for resolving sets of necklace graphs $N e_{n}$ does not hold.

Theorem 5.3.[25] For $n \geq 4, n$ even, resolving sets of the necklace graph $N e_{n}$ do not have the exchange property.

In the next theorem, we show that exchange property does not hold for resolving sets of quasi flower snarks for $n \geq 4$.

Theorem 5.4. The exchange property for minimal resolving sets does not hold in quasi flower snarks for $n \geq 4$.

Proof. We consider the following two cases.

Case (i). For $n=2 k+1$, where $k \geq 2$. Since $W=\left\{b_{0}, c_{0}, d_{n-1}\right\}$ is a metric basis [see Theorem 2.1] and hence a minimal resolving set. Also $W^{*}=\left\{a_{0}, b_{0}, c_{0}, a_{1}\right\}$ is a minimal resolving set. There is no $w \in W^{*}$ such that $S=W^{*} \backslash\{w\}$ is still a resolving set.

If $w=a_{0}$, then $r\left(a_{2 k} \mid S\right)=r\left(d_{2} \mid S\right)=(3,3,2)$. When $w=b_{0}$, then $r\left(d_{2} \mid S\right)=r\left(b_{1} \mid S\right)=(3,3,2)$. If $w=c_{0}$, then $r\left(d_{2} \mid S\right)=r\left(c_{1} \mid S\right)=(3,3,2)$ and when $w=a_{1}$, then $r\left(d_{k} \mid S\right)$ $=r\left(d_{k+1} \mid S\right)=(k+1, k+1, k+1)$. Therefore, we get $|W|=$ 3 and $\left|W^{*}\right|=4$.

Case (ii). For $n=2 k$, where $k \geq 2$. Since $W^{\prime}=\left\{a_{0}, b_{0}\right.$, $\left.c_{0}, d_{n-1}\right\}$ is a metric basis [see Theorem 2.1] and hence a 
minimal resolving set. Also $W^{*}=\left\{a_{0}, d_{0}, a_{1}, d_{k}, c_{k}\right\}$ is a minimal resolving set. There is no $w \in W^{*}$ such that $S=$ $W^{*} \backslash\{w\}$ is still a resolving set.

If $w=a_{0}$, then $r\left(a_{2 k-1} \mid S\right)=r\left(b_{1} \mid S\right)=(2,2, k, k+1)$. When $w=d_{0}$, then we get $r\left(d_{2 k-1} \mid S\right)=r\left(c_{0} \mid S\right)=(2,3, k+$ $1, k)$. If $w=a_{1}$, then $r\left(d_{1} \mid S\right)=r\left(d_{2 k-1} \mid S\right)=(2,3, k+1, k)$. If $w=d_{k}$, then $r\left(a_{2} \mid S\right)=r\left(d_{1} \mid S\right)=(2,3,1, k)$ and when $w=c_{k}$, then $r\left(b_{0} \mid S\right)=r\left(c_{0} \mid S\right)=(2,1,3, k+1)$. Therefore, $\left|W^{\prime}\right|=4$ and $\left|W^{*}\right|=5$.

There are minimal resolving sets of different size in both cases. Hence exchange property for minimal resolving sets does not hold in quasi flower snarks for $n \geq 4$. $\square$

The metric dimension of cartesian product of cycles and paths has been determined in [6] by using the idea of doubly resolving sets. In the next theorem, first we find the metric bases of cartesian product of cycles and paths by using the appropriate choice of basis vertices and then we use this result to explore the exchange property for resolving sets of cartesian product of cycles and paths.

Theorem 5.5. For every positive integer $n \geq 3$,

$$
\beta\left(C_{n} \square P_{t}\right)=\left\{\begin{array}{l}
2, \text { if } n \text { is odd; } \\
3, \text { otherwise. }
\end{array}\right.
$$

Proof. We denote $V\left(C_{n} \square P_{t}\right)=\left\{v_{i, l}: 0 \leq i \leq n-1,1 \leq l \leq\right.$ $t\}$.

Case (i). When $n=2 k+1$, where $k \geq 1$. We will show that $W=\left\{v_{0,1}, v_{k, 1}\right\}$ resolves all vertices of $C_{n} \square P_{t}$ by giving the representations of $V\left(C_{n} \square P_{t}\right) \backslash W$. For $l=1$

$r\left(v_{i, l} \mid W\right)= \begin{cases}(i, k-i), & 1 \leq i \leq k-1 ; \\ (2 k-i+1, i-k), & k+1 \leq i \leq 2 k\end{cases}$

and for $l \geq 2$, we have

$r\left(v_{i, l} \mid W\right)=\left\{\begin{array}{lr}(i+l-1, k+l-i-1), & 0 \leq i \leq k \\ (2 k+l-i, i+l-k-1), & k+1 \leq i \leq 2 k .\end{array}\right.$

It can be seen that all vertices have distinct representations. It shows that $\beta\left(C_{n} \square P_{t}\right) \leq 2$ when $n=2 k+1$, where $k \geq 1$.

Conversely, we show that $\beta\left(C_{n} \square P_{t}\right) \geq 2$. Suppose contrarily that $\beta\left(C_{n} \square P_{t}\right)=1$, but then from Theorem 1.1, a contradiction. Hence $\beta\left(C_{n} \square P_{t}\right)=2$ when $n=2 k+1$, where $k \geq 1$.

Case (ii). When $n=2 k$, where $k \geq 2$. First, we will give the representations of $V\left(C_{n} \square P_{t}\right) \backslash W$ with respect to $W$. For $l=1$

$r\left(v_{i, l} \mid W\right)=\left\{\begin{array}{lr}(i, k-i), & 1 \leq i \leq k-1 ; \\ (2 k-i, i-k), & k+1 \leq i \leq 2 k-1\end{array}\right.$

and for $l \geq 2$, we have

$r\left(v_{i, l} \mid W\right)=\left\{\begin{array}{r}(i+l-1, k+l-i-1), \quad 0 \leq i \leq k ; \\ (2 k+l-i-1, i+l-k-1), \\ k+1 \leq i \leq 2 k-1 .\end{array}\right.$

It can be seen that for $1 \leq i \leq k-1, r\left(v_{i, l} \mid W\right)=r\left(v_{2 k-i, l} \mid\right.$
$W)=(i+l-1, k+l-i-1)$. In order to have distinct representations we add the vertex $v_{1,1}$ to $W$. Then $d\left(v_{1,1}, v_{i, l}\right)=i+l-2$ and $d\left(v_{1,1}, v_{2 k-i, l}\right)=i+l$, where $1 \leq i \leq k-1$. Thus we have $W^{\prime}=W \cup\left\{v_{1,1}\right\}$ which resolves $V\left(C_{n} \square P_{t}\right)$. This mean that $\beta\left(C_{n} \square P_{t}\right) \leq 3$ for $n=2 k$, where $k \geq 2$.

Conversely, we show that $\beta\left(C_{n} \square P_{t}\right) \geq 3$ for $n=2 k$, where $k \geq 2$. Suppose that the sets of vertices $\left\{v_{0,1}, v_{1,1}\right.$, $\left.\cdots, v_{n-1,1}\right\}$ and $\left\{v_{0, t}, v_{1, t}, \cdots, v_{n-1, t}\right\}$ induce the inner and outer cycles respectively. Suppose on contrary that $\beta\left(C_{n}\right.$ $\left.\square P_{t}\right)=2$, then the following possibilities arise.

(1). If we take any of the basis vertex from the set $\left\{v_{0,2}, v_{1,2}, \cdots, v_{0,3}, v_{1,3}, \cdots, v_{n-1, t-1}\right\}$, then by Theorem 1.2, we get a contradiction.

(2). Without loss of generality, suppose that both basis vertices belong to the inner cycle. Then we may fix $v_{0,1}$ as a basis vertex. If we choose $v_{i, 1}$, where $1 \leq i \leq 2 k-1$, as a second basis vertex, then we get

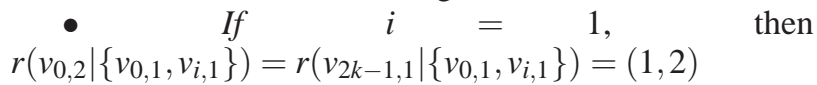

$\bullet \quad$ If $2 \leq i \leq k-1, \quad$ then $r\left(v_{i+1,1} \mid\left\{v_{0,1}, v_{i, 1}\right\}\right)=r\left(v_{i, 2} \mid\left\{v_{0,1}, v_{i, 1}\right\}\right)=(i+1,1)$

- If $i=k$, then $r\left(v_{1,1} \mid\left\{v_{0,1}, v_{i, 1}\right\}\right)=r\left(v_{2 k-1,1} \mid\left\{v_{0,1}, v_{i, 1}\right\}\right)=(1, k-1)$

- If $k+1 \leq i \leq 2 k-2$, then $r\left(v_{i-1,1} \mid\left\{v_{0,1}, v_{i, 1}\right\}\right)=r\left(v_{i, 2} \mid\left\{v_{0,1}, v_{i, 1}\right\}\right)=(2 k-i+1,1)$

- If $i=2 k-1$, then $r\left(v_{2 k-2,1} \mid\left\{v_{0,1}, v_{i, 1}\right\}\right)=r\left(v_{2 k-1,2} \mid\right.$ $\left.\left\{v_{0,1}, v_{i, 1}\right\}\right)=(2,1)$,

a contradiction in each case.

(3). When one vertex is from inner cycle and other vertex is from outer cycle. Now we may fix $v_{0,1}$ from inner cycle, if the other vertex is $v_{i, t}$ where $0 \leq i \leq 2 k-1$.

- If $i=0$, then $r\left(v_{1,1} \mid\left\{v_{0,1}, v_{i, t}\right\}\right)=r\left(v_{2 k-1,1} \mid\left\{v_{0,1}, v_{i, t}\right\}\right)=(1, t)$

- If $1 \leq i \leq k$, then $r\left(v_{i, t-1} \mid\left\{v_{0,1}, v_{i, t}\right\}\right)=r\left(v_{i-1, t} \mid\left\{v_{0,1}\right.\right.$, $\left.\left.v_{i, t}\right\}\right)=(i+t-2,1)$

- If $k+1 \leq i \leq 2 k-1$, then $r\left(v_{i, t-1} \mid\left\{v_{0,1}, v_{i, t}\right\}\right)=r$ $\left(v_{i+1, t} \mid\left\{v_{0,1}, v_{i, t}\right\}\right)=(2 k+t-i-2,1)$, where the indices are taken modulo $\mathrm{n}$.

A contradiction in all cases. Hence we have $\beta\left(C_{n} \square P_{t}\right)$ $=3$ for $n=2 k+1$, where $k \geq 2$. $\square$

In the next theorem, we study the exchange property for resolving sets in cartesian product of cycles and path (generalized prism).

Theorem 5.6. For every positive integer $n \geq 4$, resolving sets do not have exchange property in $C_{n} \square P_{t}$ for $n=2 k+1$ when $t \geq 2$ and for $n=2 k$ when $t \geq 3$.

Proof. Case (i). For $n=2 k+1$, where $k \geq 2$ and $t \geq 2$. Since $W=\left\{v_{0,1}, v_{k, 1}\right\}$ is a metric basis [see Theorem 5.5] and hence a minimal resolving set. Also $W^{*}=\left\{v_{0,1}, v_{1,1}\right.$, $\left.v_{0, t}\right\}$ is a minimal resolving set. There is no $w \in W^{*}$ such that $S=W^{*} \backslash\{w\}$ is still a resolving set.

If $w=v_{0,1}$, then $r\left(v_{0,1} \mid S\right)=r\left(v_{1,2} \mid S\right)=(1, t-1)$. When $w=v_{1,1}$, we get $r\left(v_{1,1} \mid S\right)=r\left(v_{2 k, 1} \mid S\right)=(1, t)$ and if $w=v_{0, t}$, then $r\left(v_{0, t} \mid S\right)=r\left(v_{2 k, t-1} \mid S\right)=(t-1, t)$. Therefore, $|W|=2$ and $\left|W^{*}\right|=3$. 
Case (ii). For $n=2 k$, where $k \geq 2$ and $t \geq 3$. Since $W^{\prime}=\left\{v_{0,1}, v_{k, 1}, v_{1,1}\right\}$ is a metric basis [see Theorem 5.5] and hence a minimal resolving set. Also $W^{*}=\left\{v_{0,1}, v_{1,2}\right.$, $\left.v_{k, 2}, v_{2 k-1,2}\right\}$ is a minimal resolving set. There is no $w \in$ $W^{*}$ such that $S=W^{*} \backslash\{w\}$ is still a resolving set.

If $w=v_{0,1}$, then $r\left(v_{1,1} \mid S\right)=r\left(v_{1,3} \mid S\right)=(1, k, 3)$. When $w=v_{1,2}$, then we get $r\left(v_{0,2} \mid S\right)=r\left(v_{2 k-1,1} \mid S\right)=(1, k, 1)$. If $w=v_{k, 2}$, then for $n=4$ we have $r\left(v_{2,1} \mid S\right)=r\left(v_{0,3} \mid S\right)=$ $(2,2,2)$ and for $n \geq 6$ we have $r\left(v_{2,2} \mid S\right)=r\left(v_{1,3} \mid S\right)=(3,1$, $3)$. And when $w=v_{2 k-1,2}$, we get $r\left(v_{1,1} \mid S\right)=r\left(v_{0,2} \mid S\right)=$ $(1,1, k)$. Therefore, $\left|W^{\prime}\right|=3$ and $\left|W^{*}\right|=4$.

In each case, there are minimal resolving sets of different size. Hence exchange property does not hold in $C_{n} \square P_{t}$ for $n=2 k+1$ when $t \geq 2$ and for $n=2 k$ when $t \geq 3$. $\square$

The exchange property for resolving sets of generalized antiprism $A_{n}^{m}$, where $3 \leq m \leq 5$ will be discussed in the next theorem.

Theorem 5.7. For every positive integer $n \geq 6$, resolving sets do not have exchange property in $A_{n}^{m}$ when $3 \leq m \leq 5$.

Proof. Case (i). For $n=2 k+1$, where $k \geq 3$. Since $W=$ $\left\{v_{2,1}, v_{0,\left\lfloor\frac{m-1}{2}\right\rfloor}, v_{0, m}\right\}$ is a metric basis [see Theorem 4.1] and hence a minimal resolving set. Also $W^{*}=\left\{v_{0,2}, v_{1,2}\right.$, $\left.v_{0, m}, v_{k, 2}\right\}$ is a minimal resolving set. There is no $w \in W^{*}$ such that $S=W^{*} \backslash\{w\}$ is still a resolving set.

If $w=v_{0,2}$, then $r\left(v_{k+1,1} \mid S\right)=r\left(v_{k+1,2} \mid\right.$ $S)=(k, k+1,1)$. When $w=v_{1,2}$, we get $r\left(v_{1,1} \mid S\right)=r\left(v_{2 k, 2} \mid S\right)=(1, m-1, k)$. If $w=v_{0, m}$, then $r\left(v_{1,1} \mid S\right)=r\left(v_{0,3} \mid S\right)=(1,1, k)$ and when $w=v_{k, 2}$, then $r\left(v_{0,1} \mid S\right)=r\left(v_{2 k, 2} \mid S\right)=(1,2, m-1)$. Therefore, we get $|W|=3$ and $\left|W^{*}\right|=4$.

Case (ii). For $n=2 k$, where $k \geq 3$. Since $W=\left\{v_{2,1}\right.$, $\left.v_{0,\left\lfloor\frac{m-1}{2}\right\rfloor}, v_{0, m}\right\}$ is a metric basis [see Theorem 4.1] and hence a minimal resolving set. Also $W^{*}=\left\{v_{0,1}, v_{1,2}, v_{k-1,2}\right.$, $\left.v_{2 k-1,2}\right\}$ is a minimal resolving set. There is no $w \in W^{*}$ such that $S=W^{*} \backslash\{w\}$ is still a resolving set.

If $w=v_{0,1}$, then $r\left(v_{1,1} \mid S\right)=r\left(v_{0,3} \mid S\right)=(1, k-1,2)$. When $w=v_{1,2}$, then any pair of vertices from $\left\{v_{2 k-1, m}\right.$, $\left.v_{2 k-2, m}, v_{2 k-3, m}\right\}$ have the same representation $(m-1, k$ or $k+1, m-2)$ with respect to $S$. If $w=v_{k-1,2}$, then $r\left(v_{k+1,2} \mid S\right)=r\left(v_{k+1,3} \mid S\right)=(k-1, k, k-2)$ and when $w \stackrel{1,}{=} v_{2 k-1,2}$, then we have $r\left(v_{1,1} \mid S\right)=r\left(v_{0,2} \mid S\right)=(1,1, k-1)$. Therefore, $|W|=3$ and $\left|W^{*}\right|=4$.

Since there are minimal resolving sets having different size, hence exchange property does not hold in $A_{n}^{m}$ for $n \geq$ 6 and when $3 \leq m \leq 5$.

\section{Conclusion}

The problem of determining whether $\beta(G)<k$ is an $N P$-complete problem. In this paper, we have studied the metric dimension of several classes of rotationally-symmetric graphs namely quasi flower snarks, generalized antiprism and cartesian product of square cycle with a path. It can be seen that these families of graphs have bounded or constant metric dimension, that is their metric dimensions do not depend upon the number of vertices they have. For generalized antiprism we can find the exact value of its metric dimension when $2 \leq m \leq 5$. It has been shown that exchange property does not hold for minimal resolving sets of generalized antiprism when $3 \leq m \leq 5$, generalized prism and quasi flower snarks. We close this section by raising questions that naturally arise from the text.

Open Problem 1: Determine the exact value for the metric dimension of generalized antiprism $A_{n}^{m}$ when $m \geq 6$.

Open Problem 2: Determine the exact value for the metric dimension of quasi flower snarks $G_{n}$ for $n \equiv 0$ $(\bmod 2)$.

Open Problem 3: Determine the exact value for the metric dimension of $C_{n}^{2} \square P_{t}$ when $n \equiv 1(\bmod 4)$.

\section{Acknowledgements}

This research is partially supported by NUST, Islamabad and Higher Education Commission of Pakistan. The authors are grateful to the anonymous referees for their careful checking of the details and for helpful comments that improved the first version of this paper.

\section{References}

[1] M. Ali, G. Ali, M. Imran, A. Q. Baig, M. K. Shafiq, On the metric dimension of Möbius ladders, Ars Combin., 105, 403 - 410 (2012).

[2] M. Ali, M. T. Rahim, G. Ali, On path related graphs with constant metric dimension, Utilitas Math., 88, 203 209 (2012).

[3] M. Bača, E. T. Baskoro, A. N. M. Salman, S. W. Saputro, D. Suprijanto, The metric dimension of regular bipartite graphs, Bull. Math. Soc. Sci. Math. Roumanie, 54, 141 - 153 (2011).

[4] D. L. Boutin, Determining sets, resolving sets, and the exchange property, Graphs and Combin., 25, 789 806 (2009).

[5] P. S. Buczkowski, G. Chartrand, C. Poisson, P. Zhang, On kdimensional graphs and their bases, Periodica Math. Hung., 46, $9-15$ (2003).

[6] J. Caceres, C. Hernando, M. Mora, I. M. Pelayo, M. L. Puertas, C. Seara, D. R. Wood, On the metric dimension of cartesian product of graphs, SIAM J. Disc. Math., 2, $423-$ 441 (2007).

[7] G. Chartrand, L. Eroh, M. A. Johnson, O. R. Oellermann, Resolvability in graphs and metric dimension of a graph, Disc. Appl. Math., 105, $99-113$ (2000).

[8] M. R. Garey, D. S. Johnson, Computers and Intractability: A Guide to the Theory of NP- Completeness, Freeman, New York, (1979).

[9] F. Harary, R. A. Melter, On the metric dimension of a graph, Ars Combin., 2, 191 - 195 (1976). 
[10] M. Imran, A. Ahmad, S. A. Bokhary, A. SeminičovaFeňovčikovà, On classes of regular graphs with constant metric dimension, Acta Math. Scientia, 33, 187 - 206 (2013).

[11] M. Imran, A. Q. Baig, A. Ahmad, Families of plane graphs with constant metric dimension, Utilitas Math., 88, 43 57 (2012).

[12] M. Imran, A. Q. Baig, M. K. Shafiq, I. Tomescu, On metric dimension of generalized Petersen graphs $P(n, 3)$, Ars Combin., in press.

[13] M. Imran, S. A. Bokhary, A. Q. Baig, On families of convex polytopes with constant metric dimension, Comput. Math. Appl., 60, 2629 - 2638 (2010).

[14] M. Imran, A. Q. Baig, S. A. Bokhary, I. Javaid, On the metric dimension of circulant graphs, Appl. Math. Letters, 25, 320 - 325 (2012).

[15] H. Iswadi, E. T. Baskoro, R. Simanjuntak, A. N. M. Salman, The metric dimension of graphs with pendent edges, $J$. Combin. Math. Combin. Comput., 65, 139 - 146 (2008).

[16] I. Javaid, M. T. Rahim, K. Ali, Families of regular graphs with constant metric dimension, Utilitas Math., 75, 21 33 (2008).

[17] S. Khuller, B. Raghavachari, A. Rosenfeld, Landmarks in graphs, Disc. Appl. Math., 70, 217 - 229 (1996).

[18] S. Khuller, B. Raghavachari, A. Rosenfeld, Localization in graphs, Technical Report CS-TR-3326, University of Maryland at College Park, (1994).

[19] R. A. Melter, I. Tomescu, Metric bases in digital geometry, Computer Vision, Graphics, and Image Processing, 25, 113 121 (1984).

[20] S. W. Saputro, R. Simanjuntak, S. Uttunggadewa, H. Assiyatun, E. T. Baskoro, A. N. M. Salman, M. Bača, The metric dimension of the lexicographic product of graphs, Disc. Math., 313, 1045 - 1051 (2013).

[21] A. Sebö, E. Tannier, On metric generators of graphs, Math. Oper. Res., 29, 383 - 393 (2004).

[22] P. J. Slater, Leaves of trees, Congress. Numer., 14, $549-$ 559 (1975).

[23] I. Tomescu, M. Imran, On metric and partition dimensions of some infinite regular graphs, Bull. Math. Soc. Sci. Math. Roumanie, 52, 461 - 472 (2009).

[24] I. Tomescu, M. Imran, Metric dimension and $R$-sets of connected graphs, Graphs and Combin., 27,585591(2011).

[25] I. Tomescu, M. Imran, $R$-sets and metric dimension of necklace graphs, Appl. Math. Inf. Sci., (in press).

[26] I. Tomescu, I. Javaid, On the metric dimension of the Jahangir graph, Bull. Math. Soc. Sci. Math. Roumanie, 50, $371-376$ (2007).

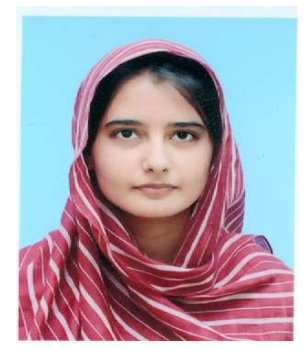

Rishi Naeem is a $\mathrm{PhD}$ student of Mathematics at the School of Natural Sciences (SNS), National University of Sciences and Technology (NUST), Islamabad, Pakistan. She completed her M. Phil in Mathematics in 2013. Currently, she is a $\mathrm{PhD}$ student at the same School. analysis and graph theory. Her research interests include

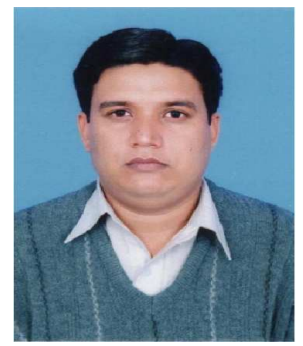

Muhammad Imran is an Assistant Professor of Mathematics at the School of Natural Sciences (SNS), National University of Sciences and Technology (NUST), Islamabad, Pakistan. His research interests include metric graph theory, graph labeling and spectral graph theory. He has published research articles in reputed international journals of Mathematics and Informatics. He is referee of several international mathematical journals. 